[4] Hansen, K.F., Sakamoto, K., Obrietan, K., 2011. MicroRNAs: a potential interface between the circadian clock and human health. Genom Med 3, 10-17.

Disclosure statement: The study was supported by the MTA-SE-NAP B Genetic Brain Imaging Migraine Research Group (KTIA_NAP_13-22015-0001) by the National Development Agency (KTIA NAP 13-12013-0001), Hungarian Brain Research Program (KTIA_13_NAP-A-II/ 14) and the MTA-SE Neuropsychopharmacology and Neurochemistry Research Group.

\section{P.1.a.005 Genome-wide gene-based tests replicate the association of the SORCS3 gene with neuroticism}

N. Eszlari ${ }^{1 *}$, A. Millinghoffer ${ }^{2}$, P. Petschner ${ }^{1}$, X. Gonda ${ }^{3}$, D. Baksa ${ }^{4}$, A.J. Pulay ${ }^{5}$, J. Rethelyi ${ }^{6}$, R. Elliott ${ }^{7}$, I.M. Anderson ${ }^{7}$ J.F.W. Deakin ${ }^{7}$, P. Antal ${ }^{2}$, G. Bagdy ${ }^{1}$, G. Juhasz ${ }^{1}{ }^{1}$ Semmelweis University, Department of Pharmacodynamics, Budapest, Hungary; ${ }^{2}$ Budapest University of Technology and Economics, Department of Measurement and Information Systems, Budapest, Hungary; ${ }^{3}$ Semmelweis University, Department of Psychiatry and Psychotherapy, Kutvolgyi Clinical Centre, Budapest, Hungary; ${ }^{4}$ Hungarian Academy of Sciences, Semmelweis University, MTA-SE-NAP B Genetic Brain Imaging Migraine Research Group, Budapest, Hungary; ${ }^{5}$ Semmelweis University, Department of Psychiatry and Psychotherapy, Budapest, Hungary;

${ }^{6}$ Hungarian Academy of Sciences, Semmelweis University, MTA-SE NAP-B Molecular Psychiatry Research Group, Budapest, Hungary; ${ }^{7}$ University of Manchester, Institute of Brain Behaviour and Mental Health, Neuroscience and Psychiatry Unit,

Manchester, United Kingdom

Introduction: Among the Big Five personality dimensions, neuroticism has the strongest phenotypic as well as genotypic correlations with lifetime major depression [1]. Previous genomewide association studies (GWAS) have applied SNP (single nucleotide polymorphism)-based and gene-based tests to find SNPs and genes in the background of neuroticism. However, in those GWAS implementing gene-based tests, only one method was used.

Aim: In the present study, we conducted a GWAS on neuroticism, both with SNP-based and with five distinct genebased tests.

Methods: Among a European white sample $(\mathrm{N}=1770)$ recruited in Budapest and Manchester, linear regression models were run with each of the genome-wide genotyped 86,232 SNPs, gender, age and the top ten principal components of the genome as predictor variables, and neuroticism as the outcome. The yielded SNP-based results then served as the input of the gene-based GATES test. False discovery rate (FDR) method was used to correct for multiple testing both at the SNP- and the gene-level, with an error rate of 0.05 . As post hoc tests without correction, four additional gene-based tests were implemented according to FORGE and SETSCREEN methods.
Results: 63,326 SNPs (64.46\% of all) reside within genes, and 18,264 genes were identified. None of the SNPs or genes survives the FDR correction. However, among the more than fifty genes identified as candidates in previous neuroticism GWAS studies either by a gene-based test or by locating any significant SNP into a gene, we found that TACC2 (transforming acidic coiled-coil containing protein 2 ) and SORCS3 (sortilin related VPS10 domain containing receptor 3 ) genes are nominally significant $(\mathrm{p} \leq 0.05)$ to neuroticism in our study (Table 1). Nonetheless, TACC2 does not show any significant $(\mathrm{p} \leq 0.05)$ association with neuroticism according to our post hoc gene-based tests, but the effect of SORCS3 is significant in all of the four tests (Table 1).

Conclusion: Our GWAS study is the first one to report the genelevel association of the SORCS3 gene with neuroticism, since the original GWAS from which we chose this gene for replication did not use a gene-based, but only a SNP-based approach [2]. Moreover, we further underpinned this gene-personality association with four additional methods. SORCS3 SNPs have been reported to be associated with Alzheimer's disease, and the expression level of this gene was lower in Alzheimer's patients' than in controls' amygdala, but there was no such between-group difference in occipital lobe or cerebellum [3]. Given the wellgrounded importance of the amygdala in depression risk and neuroticism, further studies are needed to elucidate the precise role of the SORCS3 gene and SORCS3 protein in neuroticism and depression, and to reveal whether or not their association with Alzheimer's disease is mediated by this personality trait.

\section{References}

[1] Kendler, K.S., Myers, J., 2010. The genetic and environmental relationship between major depression and the five-factor model of personality. Psychol Med 40, 801-806.

[2] Bae, H.T., Sebastiani, P., Sun, J.X., Andersen, S.L., Daw, E.W., Terracciano, A., Ferrucci, L., Perls, T.Z., 2013. Genome-wide association study of personality traits in the Long Life Family Study. Front Genet 8 (4), 65

[3] Reitz, C., Tosto, G., Vardarajan, B., Rogaeva, E., Ghani, M., Rogers, R.S., Conrad, C., Haines, J.L., Pericak-Vance, M.A., Fallin, M.D., Foroud, T., Farrer, L.A., Schellenberg, G.D., GeorgeHyslop, P.S., Mayeux, R.; the Alzheimer's Disease Genetics Consortium (ADGC), 2013. Independent and epistatic effects of variants in VPS10-d receptors on Alzheimer disease risk and processing of the amyloid precursor protein (APP). Transl Psychiatry 3, e256. doi: $10.1038 /$ tp.2013.13

Disclosure statement: Acknowledgements: The study was supported by the Sixth Framework Program of the European Union, NewMood, LSHM-CT-2004-503474 by the National Institute for Health Research Manchester Biomedical Research Centre by the Hungarian Brain Research Program (Grant KTIA_13_NAP-A-II/14) and the National Development Agency (Grant KTIA NAP 13-1-2013-0001) by the Hungarian Academy of Sciences (MTA-SE Neuropsychopharmacology and Neurochemistry Research Group) by the Hungarian Academy of Sciences and the Hungarian Brain Research Program - Grant No. KTIA_NAP_13-2-2015-0001 (MTA-SE-NAP B Genetic Brain Imaging Migraine Research Group) and by the ÚNKP-16-3 New National

Table 1. (Abstract P.1.a.005) Gene-based methods to assess the association of neuroticism with two genes reported in previous GWAS.

\begin{tabular}{|c|c|c|c|c|c|c|}
\hline \multirow[b]{2}{*}{ Gene } & \multirow{2}{*}{$\frac{\text { GATES method }}{\text { Nominal P value }}$} & \multicolumn{5}{|c|}{ Post hoc tests } \\
\hline & & FDR-corrected P value & FORGE PSidak & FORGE Zfix & SETSCREEN P1 & SETSCREEN P2 \\
\hline TACC2 & 0.0449 & 0.9696 & 0.9089 & 0.9857 & 0.9599 & 0.9698 \\
\hline SORCS3 & 0.0130 & 0.9296 & 0.0201 & 0.0106 & 0.0177 & 0.0437 \\
\hline
\end{tabular}


Excellence Program of The Ministry of Human Capacities. Xenia Gonda and Peter Antal are recipients of the Janos Bolyai Research Fellowship of the Hungarian Academy of Sciences. We thank Diana Chase, Emma J. Thomas, Darragh Downey, Kathryn Lloyd-Williams and Zoltan G. Toth for their assistance in the recruitment and data acquisition and the Heaton Mersey Medical Practice and Cheadle Medical Practice for their assistance in the recruitment. We thank Charles Curtis for his assistance in genotyping (King's College London, SGDP Centre, Institute of Psychiatry). Conflict of interest: John Francis William Deakin has variously performed consultancy, speaking engagements and research for Bristol-Myers Squibb, AstraZeneca, Eli Lilly, Schering Plough, JanssenCilag and Servier (all fees are paid to the University of Manchester to reimburse them for the time taken) he also has share options in P1vital. Ian Muir Anderson has received consultancy fees from Servier, Alkermes, Lundbeck/Otsuka and Janssen, an honorarium for speaking from Lundbeck and grant support from Servier and AstraZeneca. Rebecca Elliott has received consultancy fees from Cambridge Cognition and P1vital. The remaining authors declare no conflict of interest.

\section{P.1.a.006 Association of dopamine transporter gene SLC6A3 polymorphisms with remission and antidepressant therapy response in patients with depressive disorders}

I.S. Losenkov ${ }^{1 *}$ I.V. Pozhidaev ${ }^{2}$, D.Z. Osmanova ${ }^{2}$, G.G. Simutkin ${ }^{3}{ }^{1}$ Mental Health Research Institute, Tomsk National Research Medical Center, Russian Academy of Sciences, Laboratory of Molecular Genetics and Biochemistry, Tomsk, Russia; ${ }^{2}$ Tomsk State University, Department of Cytology and Genetics, Tomsk, Russia; ${ }^{3}$ Mental Health Research Institute, Tomsk National Research Medical Center, Russian Academy of Sciences, Department of Affective Disorders, Tomsk, Russia

Background: Nowadays the problem of antidepressant therapy in unipolar depression is considered important and the question of treatment and prophylactics is extensively discussed in psychiatry. Up-to-date information about biological markers which allows to predict therapy response, remission and side effects is not numerous $[1,2]$. The latest data consider the role of dopamine transporter DAT1 coded by SLC6A3 gene in the pathogenesis of depressive disorders, but results remain inconsistent $[3,4]$. In view of these recent data SLC6A3 gene is a possible candidate for pharmacogenetics studies.

Aim of the study: The aim of the research was to investigate the association of single nucleotide polymorphisms (SNPs) of SLC6A3 gene, encoding dopamine transporter DAT1, with remission and antidepressant therapy response in patients with depressive disorders.

Materials and methods: 171 patients (96 patients with depressive episode (ICD-10: F32), 75 patients with recurrent depressive disorder (ICD-10: F33), 35 male and 136 female in age between 20 and 60 years) were included in the study. Antidepressant therapy response on the 14th and 28th day of therapy was evaluated using Clinical Global Impression Improvement scale (CGI-I). Remission was assessed by Hamilton Depressive Rating Scale 17 (HDRS-17): score $\leq 7$ for remitters. 12 SNPs (rs3756450, rs2550956, rs6347, rs2617605, rs3863145, rs250686, rs464049, rs4975646, rs1048953, rs11133767, rs27048, rs40184) of SLC6A3 gene were genotyped. The chi-squared test was used for Hardy-Weinberg equilibrium and for between-groups of remitters and non-remitters comparison of genotypic and allelic frequencies. Median and quartiles for CGI-I scale scores were calculated. Between-group differences were evaluated using Kruskal-Wallis H test. Statistical analyses were performed using the SPSS software, release 20.0, for Windows. $p$ values less than 0.05 were considered as significant.

Results: Correlation between CGI-I scale scores and SNPs of SLC6A3 gene were studied. Patients with genotype T/T of SNP rs464049 had a higher score of CGI-I scale on 28th day of therapy (2.0 (1.0-2.0)) compared to patients with $\mathrm{C} / \mathrm{C}(1.0$ (1.0 $2.0))$ and $C / T(1.0(1.0-2.0))$ genotypes $(p=0.045)$. The highest score of CGI-I scale on 28th day of treatment $(2.0$ (1.0-2.0)) was found in patients with T/T genotype of SNP $\mathrm{rs} 1048953$ ( $\mathrm{p}=$ 0.011). Genotypic and allelic frequencies of SNPs of SLC6A3 gene in groups of remitters and non-remitters were studied. Distribution of genotypes was in agreement with HardyWeinberg equilibrium. Genotype C/C of SNP rs2550956 was not observed in the group of non-remitters and its frequency in the group of remitters was $9.3 \%(\mathrm{p}=0.027 ; \mathrm{OR}=8.93,95 \% \mathrm{Cl}$ $=0.52-152.39$ ). Frequency of $\mathrm{G} / \mathrm{G}$ genotype of SNP rs6347 was higher in the group of non-remitters $(19.1 \%)$ compared to the group of remitters $(6.9 \%)(\mathrm{p}=0.041 ; \mathrm{OR}=3.19,95 \% \mathrm{Cl}=$ 1.19-8.53). Frequency of allele $\mathrm{G}$ was higher in the group of non-remitters $(23.8 \%)$ comparing to the group of remitters $(15.0 \%)(\mathrm{p}=0.050 ; \mathrm{OR}=1.77,95 \% \mathrm{Cl}=0.98-3.19)$.

Conclusion: We have found an association between SNPs rs464049, rs1048953 and antidepressant therapy response. Asscociation of SNPs rs2550956, rs6347 and remission has been observed. It is possible that SNPs rs464049, rs 1048953 and rs6347 are associated with blunted therapy response.

\section{References}

[1] Bauer, M., Pfennig, A., Severus, E., Whybro, P.C., Angst, J., Möller, H. J., 2013. World Federation of Societies of Biological Psychiatry (WFSBP) guidelines for biological treatment of unipolar depressive disorders. Part 1: update 2013 on the acute and continuation treatment of unipolar depressive disorders. World J Biol Psychiatry 14, 334-385.

[2] Bauer, M., Severus, E., Whybro, P.C., Angst, J., Möller, H.J., 2015. World Federation of Societies of Biological Psychiatry (WFSBP) guidelines for biological treatment of unipolar depressive disorders. Part 2: maintenance treatment of major depressive disorder-update. World J Biol Psychiatry 16, 76-95.

[3] Camardese, G., Di Giuda, D., Di Nicola, M., Cocciolillo, F., Giordano, A., Janiri, L., Guglielmo, R., 2014. Imaging studies on dopamine transporter and depression: a review of literature and suggestions for future research. J Psychiatr Res 51, 7-8.

[4] Li, Z., He, Y., Tang, J., Zong, X., Hu, M., Chen, X., 2014. Molecular imaging of striatal dopamine transporters in major depression-a metaanalysis. J Affect Disord 174, 137-143.

Disclosure statement: This work was supported by the Russian Science Foundation, grant №14-35-00023 «Laboratory of pharmacogenetic research on personalized therapy of mental and neurodegenerative disorders».

\section{P.1.a.007 Gene expression biomarkers related to auditory hallucinations in peripheral blood of patients with schizophrenia}

J. Gilabert-Juan ${ }^{1}$, G. Lopez-Campos ${ }^{2}$, N. Sebastia-Ortega ${ }^{3}$, S. Guara ${ }^{3}$, J. Sanjuan ${ }^{4}$, M.D. Molto ${ }^{3}{ }^{1}$ Hoppital Robert Debré, U1141, Paris, France; ${ }^{2}$ University of Melbourne, Health and Biomedical Informatics Centre, Melbourne, Australia; ${ }^{3}$ University of Valencia. CIBERSAM. INCLIVA, Genetics, Valencia, Spain; ${ }^{4}$ University of Valencia. CIBERSAM. INCLIVA, Medicine, Valencia, Spain

Schizophrenia is a severe mental disorder affecting around $1 \%$ of the population. This disease has a complex aetiology, involving 